\title{
An accelerated solution for some classes of nonlinear partial differential equations
}

\author{
I. L. El-Kalla', E. M. Mohamed ${ }^{2 *}$ (D) and H. A. A. El-Saka ${ }^{2}$
}

*Correspondence:

emoo_1091@yahoo.com

${ }^{2}$ Mathematics Department,

Faculty of Science, Damietta

University, New Damietta,

Egypt

Full list of author information

is available at the end of the

article

\begin{abstract}
In this paper, we apply an accelerated version of the Adomian decomposition method for solving a class of nonlinear partial differential equations. This version is a smart recursive technique in which no differentiation for computing the Adomian polynomials is needed. Convergence analysis of this version is discussed, and the error of the series solution is estimated. Some numerical examples were solved, and the numerical results illustrate the effectiveness of this version.
\end{abstract}

Keywords: Adomian decomposition method, Adomian polynomials, Nonlinear partial differential equations

Mathematics Subject Classification: 35A01, 35A02, 35A35

\section{Background}

Many physics and engineering problems are modeled by partial differential equations (PDEs). In many instances, these equations are nonlinear and the exact solutions are difficult to be obtained. Several methods were developed over some time to find approximate solutions to these nonlinear equations, such as homotopy analysis method (HAM) [1-4], homotopy perturbation method (HPM) $[1,5,6]$, and Adomian decomposition method (ADM) [7-15]. In this paper, we introduce an accelerated version of the ADM for solving some classes of NPDEs. In ADM, the nonlinear term is replaced by a series of what are called Adomian polynomials which were introduced by Adomian and his colleagues have so far. Some other authors have suggested different formulas for computing Adomian polynomials [16-23]. This work aims to apply the accelerated formula proposed by El-Kalla in [21] for solving some classes of nonlinear partial differential equations. The main advantages of this accelerated version of Adomian polynomials can be summarized in the following main three points:

1. It is recursive and does not have derivative terms so, it is easy in programming, and, on the same processor, it saves time compared with the traditional formula;

2. Solution using it converges faster than the traditional Adomian polynomials;

3. It is used in convergence analysis and consequently in estimating the maximum absolute truncated error of the series solution. author(s) and the source, provide a link to the Creative Commons licence, and indicate if changes were made. The images or other third party material in this article are included in the article's Creative Commons licence, unless indicated otherwise in a credit line to the material. If material is not included in the article's Creative Commons licence and your intended use is not permitted by statutory regulation or exceeds the permitted use, you will need to obtain permission directly from the copyright holder. To view a copy of this licence, visit http:// creativecommons.org/licenses/by/4.0/. 
The paper is organized as follows. In "The method" section, the standard ADM and the accelerated version of ADM are introduced. In "Convergence analysis" section, the convergence analysis of the accelerated version is introduced, while in "Numerical examples" section, some examples are solved to illustrate the effectiveness of this version.

\section{The method}

Consider the nonlinear partial differential equation given in the operator form:

$$
L_{t} u(x, t)+R(u(x, t))+N(u(x, t))=g(x, t),
$$

where $L_{t}()=.\frac{\partial^{k}(.)}{\partial t^{k}}, R$ is the linear remainder operator that could include partial derivatives with respect to $x, N$ is the nonlinear operator, and $g$ is the nonhomogeneous term.

Put (1) in the following form

$$
L_{t} u(x, t)=g(x, t)-R(u(x, t))-N(u(x, t)) .
$$

Applying $L_{t}^{-1}$ to both sides of (2), we obtain

$$
u(x, t)=\Phi(x, t)+L_{t}^{-1} g(x, t)-L_{t}^{-1} R(u(x, t))-L_{t}^{-1} N(u(x, t)),
$$

where $\Phi(x, t)$ is the solution of $L_{t} u(x, t)=0$ satisfied by the given initial conditions and $L_{t}^{-1}()=.\int_{0}^{t} \ldots k$-fold $\ldots \int_{0}^{t}(.) \mathrm{d} t \ldots \mathrm{d} t$.

ADM assumes that the solution $u$ can be decomposed into infinite series

$$
u(x, t)=\sum_{n=0}^{\infty} u_{n}(x, t)
$$

and the nonlinear term $\mathrm{Nu}$ by:

$$
N u=\sum_{n=0}^{\infty} A_{n} .
$$

The components $u_{n}, n \geq 0$ of the solution $u$ can be determined by using the recursive relation:

$$
\begin{aligned}
u_{0} & =\Phi(x, t)+L_{t}^{-1} g(x, t), \\
u_{n+1} & =-L_{t}^{-1}\left(R u_{n}\right)-L_{t}^{-1}\left(A_{n}\right), \quad n \geq 0,
\end{aligned}
$$

where $A_{n}=A_{n}\left(u_{0}, u_{1}, \ldots, u_{n}\right)$ is Adomian polynomials that can be determined by the traditional polynomials formula, 


$$
A_{n}=\frac{1}{n !} \frac{\mathrm{d}^{n}}{\mathrm{~d} \lambda^{n}}\left[N\left(\sum_{i=0}^{\infty} \lambda^{i} u_{i}\right)\right]_{\lambda=0}, \quad n=0,1,2, \ldots
$$

or by El-Kalla formula [21],

$$
\bar{A}_{n}=N\left(S_{n}\right)-\sum_{i=0}^{n-1} \bar{A}_{i}, \quad n=0,1,2, \ldots,
$$

where the partial sum $S_{n}=\sum_{i=0}^{n} u_{i}(x, t)$.

For example, Table 1 shows the first four polynomials of the nonlinear term $u^{2}$ generated by both the traditional polynomials formula (7) and El-Kalla polynomials formula (8).

Clearly, the first four polynomials generated by El-Kalla formula (8) include the first four polynomials generated by the traditional formula (7) in addition to other terms that should appear in $A_{4}, A_{5}, \ldots$ using formula (7). Thus, the solution obtained using El-Kalla polynomials converges faster than the solution obtained using the traditional polynomials.

\section{Convergence analysis}

Theorem 1 (Uniqueness theorem) Assume that $R$ and $N$ are Lipschitzian with respect to $u$ such that $|R(u)-R(v)| \leq L_{1}|u-v|$ and $|N(u)-N(v)| \leq L_{2}|u-v|$. Let $E=(C[\Omega],\|\cdot\|)$ denote the Banach space of all continuous functions on the domain of $x$ and $t ; \Omega=[0, x] \times[0, T]$ with the norm $\|u(x, t)\|=\max _{\Omega}|u(x, t)|$. Then, problem (1) has a unique solution whenever $0<\alpha<1, \alpha=\frac{\left(L_{1}+L_{2}\right) T^{k}}{k !}$.

Proof Define a mapping $F: E \rightarrow E$ such that $F(u)=\Phi(x, t)+L_{t}^{-1} g(x, t)-L_{t}^{-1} R(u(x, t))-L_{t}^{-1} N(u(x, t))$ and let $u, u^{*} \in E$. Then,

Table 1 The first four Adomian polynomials and the first four El-Kalla polynomials of the nonlinear term $u^{2}$

\begin{tabular}{ll}
\hline Adomian polynomials of $u^{\mathbf{2}}$ & El-Kalla polynomials of $u^{\mathbf{2}}$ \\
\hline$A_{0}=u_{0}^{2}$ & $\bar{A}_{0}=u_{0}^{2}$ \\
$A_{1}=2 u_{0} u_{1}$ & $\bar{A}_{1}=2 u_{0} u_{1}+u_{1}^{2}$ \\
$A_{2}=u_{1}^{2}+2 u_{0} u_{2}$ & $\bar{A}_{2}=2 u_{0} u_{2}+2 u_{1} u_{2}+u_{2}^{2}$ \\
$A_{3}=2 u_{1} u_{2}+2 u_{0} u_{3}$ & $\bar{A}_{3}=2 u_{0} u_{3}+2 u_{1} u_{3}+2 u_{2} u_{3}+u_{3}^{2}$ \\
\hline
\end{tabular}




$$
\begin{aligned}
\left\|F u-F u^{*}\right\| & =\max _{\Omega}\left|L_{t}^{-1}\left(R u-R u^{*}\right)+L_{t}^{-1}\left(N u-N u^{*}\right)\right| \\
& \leq \max _{\Omega} L_{t}^{-1}\left(L_{1}\left|u-u^{*}\right|+L_{2}\left|u-u^{*}\right|\right) \\
& \leq\left(L_{1}+L_{2}\right) \max _{\Omega}\left|u-u^{*}\right| \int_{0}^{t} \ldots k \text {-fold } \ldots \int_{0}^{t} \mathrm{~d} t \ldots \mathrm{d} t \\
& \leq \frac{\left(L_{1}+L_{2}\right) T^{k}}{k !}\left\|u-u^{*}\right\| \\
& \leq \alpha\left\|u-u^{*}\right\| .
\end{aligned}
$$

Under the condition $0<\alpha<1$, the mapping $F$ is contraction; therefore, by the Banach fixed-point theorem for contraction, there exists a unique solution to problem (1).

Theorem 2 (Convergence theorem) The series solution (4) of problem (1) using ADM converges whenever $0<\alpha<1$ and $|u(x, t)|<\infty$ on $\Omega$.

Proof Let, $S_{n}$ and $S_{m}$ be arbitrary partial sums with $n>m$. We are going to prove that $\left\{S_{n}\right\}$ is a Cauchy sequence in Banach space $E$. From Theorem 1, we write

$$
\left\|S_{m+1}-S_{m}\right\| \leq \alpha\left\|S_{m}-S_{m-1}\right\| \leq \alpha^{2}\left\|S_{m-1}-S_{m-2}\right\| \leq \cdots \leq \alpha^{m}\left\|S_{1}-S_{0}\right\| .
$$

Using the triangle inequality, we have

$$
\begin{aligned}
\left\|S_{n}-S_{m}\right\| & \leq\left\|S_{m+1}-S_{m}\right\|+\left\|S_{m+2}-S_{m+1}\right\|+\cdots+\left\|S_{n}-S_{n-1}\right\| \\
& \leq\left[\alpha^{m}+\alpha^{m+1}+\cdots+\alpha^{n-1}\right]\left\|S_{1}-S_{0}\right\| \\
& \leq \alpha^{m}\left[1+\alpha+\alpha^{2}+\cdots+\alpha^{n-m-1}\right]\left\|S_{1}-S_{0}\right\| \\
& \leq \alpha^{m}\left(\frac{1-\alpha^{n-m}}{1-\alpha}\right)\left\|u_{1}(x, t)\right\| .
\end{aligned}
$$

Since $0<\alpha<1$ so, $1-\alpha^{n-m}<1$, then

$$
\left\|S_{n}-S_{m}\right\| \leq \frac{\alpha^{m}}{1-\alpha} \max _{\Omega}\left|u_{1}(x, t)\right|
$$

but $\max _{\Omega}\left|u_{1}(x, t)\right|<\infty$ then $\left\|S_{n}-S_{m}\right\| \rightarrow \infty$ as $m \rightarrow \infty$, then we conclude that $\left\{S_{n}\right\}$ is a Cauchy sequence in $E$ and the series $\sum_{i=0}^{\infty} u_{i}(x, t)$ converges to the unique solution $u(x, t)$.

Theorem 3 (Error estimate) An estimate for the truncation error of the series solution (4) to problem (1) is given by:

$$
\max _{\Omega}\left|u(x, t)-\sum_{i=0}^{m} u_{i}(x, t)\right| \leq \frac{\alpha^{m}}{1-\alpha} \max _{\Omega}\left|u_{1}(x, t)\right| .
$$

Proof From (9) in Theorem 2, we have

$$
\left\|S_{n}-S_{m}\right\| \leq \frac{\alpha^{m}}{1-\alpha} \max _{\Omega}\left|u_{1}(x, t)\right|
$$


as $n \rightarrow \infty$ then $S_{n} \rightarrow u(x, t)$ so, we have

$$
\left\|u(x, t)-S_{m}\right\| \leq \frac{\alpha^{m}}{1-\alpha} \max _{\Omega}\left|u_{1}(x, t)\right| .
$$

Finally, the truncation error in the region $\Omega$ is estimated to be

$$
\max _{\Omega}\left|u(x, t)-\sum_{i=0}^{m} u_{i}(x, t)\right| \leq \frac{\alpha^{m}}{1-\alpha} \max _{\Omega}\left|u_{1}(x, t)\right| .
$$

\section{Numerical examples}

In this section, we present some numerical examples to illustrate the effectiveness of the proposed version of ADM. All the results are calculated using Mathematica 11.

Example 1 Consider the following nonlinear partial differential equation:

$$
\frac{\partial u}{\partial t}+u u_{x}=x+x t^{2}
$$

with initial condition

$$
u(x, 0)=0
$$

which has exact solution $u(x, t)=x t$.

Solution Equation (10) is rewritten in the form:

$$
L_{t} u=x+x t^{2}-u u_{x}
$$

where $N u=u u_{x}$ and $L_{t}=\frac{\partial}{\partial t}$.

$$
\begin{aligned}
& \text { Applying } L_{t}^{-1}(.)=\int_{0}^{t}(.) \mathrm{d} t \text { to (12), we get } \\
& \quad u=u(x, 0)+L_{t}^{-1}\left(x+x t^{2}\right)-L_{t}^{-1}(N u) .
\end{aligned}
$$

Based on the recurrence relation (6) and substituting the initial value, we get

$$
\begin{aligned}
& u_{0}=x t+\frac{1}{3} x t^{3}, \\
& u_{n+1}=-L_{t}^{-1}\left(A_{n}\right), n \geq 0,
\end{aligned}
$$

using the traditional polynomials formula (7),

$$
\begin{aligned}
& A_{0}=u_{0} u_{0 x}, \\
& A_{1}=u_{0} u_{1 x}+u_{1} u_{0 x}, \\
& A_{2}=u_{0} u_{2 x}+u_{1} u_{1 x}+u_{2} u_{0 x} .
\end{aligned}
$$

Then, from (14) and (15) we get 


$$
\begin{aligned}
& u_{0}=x t+\frac{1}{3} x t^{3}, \\
& u_{1}=-\frac{t^{3} x}{3}-\frac{2 t^{5} x}{15}-\frac{t^{7} x}{63} \\
& u_{2}=\frac{17 t^{6} x}{45}+\frac{20 t^{8} x}{63}+\frac{206 t^{10} x}{2025}+\frac{1412 t^{12} x}{93555}+\frac{13 t^{14} x}{14553}
\end{aligned}
$$

and using El-Kalla polynomials formula (8),

$$
\begin{aligned}
& \bar{A}_{0}=u_{0} u_{0 x}, \\
& \bar{A}_{1}=u_{0} u_{1 x}+u_{1} u_{0 x}+u_{1} u_{1 x}, \\
& \bar{A}_{2}=u_{0} u_{2 x}+u_{1} u_{2 x}+u_{2} u_{0 x}+u_{2} u_{1 x}+u_{2} u_{2 x} .
\end{aligned}
$$

Then, from (14) and (17) we get

$$
\begin{aligned}
& u_{0}=x t+\frac{1}{3} x t^{3}, \\
& u_{1}=-\frac{t^{3} x}{3}-\frac{2 t^{5} x}{15}-\frac{t^{7} x}{63}, \\
& u_{2}=\frac{2 t^{5} x}{15}+\frac{17 t^{7} x}{315}+\frac{2 t^{9} x}{567}-\frac{4 t^{11} x}{2475}-\frac{4 t^{13} x}{12285}-\frac{t^{15} x}{59535} .
\end{aligned}
$$

Table 2 shows the absolute relative error (ARE) for the sixth-order approximate solution using the proposed version of $\mathrm{ADM}$ and the seventh-order approximate solution using the standard ADM at $t=1$ for some values of $x$ in Example 1 .

Example 2 Consider a nonlinear partial differential equation:

$$
\frac{\partial^{2} u}{\partial t^{2}}-\frac{\partial^{2} u}{\partial x^{2}}+\frac{\pi^{2}}{4} u+u^{2}=x^{2} \sin ^{2} \frac{\pi t}{2},
$$

Table 2 The absolute relative error for Example 1

\begin{tabular}{lll}
\hline & (ARE) of solution using the standard ADM & $\begin{array}{l}\text { (ARE) of solution using } \\
\text { the proposed version } \\
\text { of ADM }\end{array}$ \\
\hline$x$ & $t=1$ & $3.27534 \times 10^{-6}$ \\
0.1 & $1.3965 \times 10^{-4}$ & $6.55068 \times 10^{-6}$ \\
0.2 & $2.79299 \times 10^{-4}$ & $9.82602 \times 10^{-6}$ \\
0.3 & $4.18949 \times 10^{-4}$ & $1.31014 \times 10^{-5}$ \\
0.4 & $5.58599 \times 10^{-4}$ & $1.63767 \times 10^{-5}$ \\
0.5 & $6.98249 \times 10^{-4}$ & $1.9652 \times 10^{-5}$ \\
0.6 & $8.37898 \times 10^{-4}$ & $2.29274 \times 10^{-5}$ \\
0.7 & $9.77548 \times 10^{-4}$ & $2.62027 \times 10^{-5}$ \\
0.8 & $1.1172 \times 10^{-3}$ & $2.94781 \times 10^{-5}$ \\
0.9 & $1.25685 \times 10^{-3}$ & $3.27534 \times 10^{-5}$ \\
1 & $1.3965 \times 10^{-3}$ & \\
& The time elapsed of the program that calculates the solution & \\
& $5.3183 \mathrm{~s}$ & $3.6548 \mathrm{~s}$ \\
\hline
\end{tabular}


Table 3 The absolute relative error for Example 2

\begin{tabular}{lll}
\hline & $\begin{array}{l}\text { (ARE) of solution using the standard ADM } \\
x\end{array}$ & $\begin{array}{l}\text { (ARE) of solution using } \\
\text { the proposed version } \\
\text { of ADM }\end{array}$ \\
0.1 & $3.71362 \times 10^{-11}$ & $2.08449 \times 10^{-11}$ \\
0.2 & $2.48603 \times 10^{-11}$ & $1.21807 \times 10^{-11}$ \\
0.3 & $8.57263 \times 10^{-12}$ & $2.654 \times 10^{-12}$ \\
0.4 & $1.19764 \times 10^{-11}$ & $7.77757 \times 10^{-12}$ \\
0.5 & $3.70427 \times 10^{-11}$ & $1.91576 \times 10^{-11}$ \\
0.6 & $6.68882 \times 10^{-11}$ & $3.15307 \times 10^{-11}$ \\
0.7 & $1.01782 \times 10^{-10}$ & $4.49427 \times 10^{-11}$ \\
0.8 & $1.41997 \times 10^{-10}$ & $5.94405 \times 10^{-11}$ \\
0.9 & $1.87817 \times 10^{-10}$ & $7.50722 \times 10^{-11}$ \\
1 & $2.3953 \times 10^{-10}$ & $9.18868 \times 10^{-11}$ \\
& The time elapsed of the program that calculates the solution & \\
& $9.7154 \mathrm{~s}$ & $4.5263 \mathrm{~s}$ \\
\hline
\end{tabular}

with initial condition

$$
u(x, 0)=0, u_{t}(x, 0)=\frac{\pi x}{2} .
$$

This problem was solved in [24] by using the standard Adomian decomposition method. Now, we will apply the proposed accelerated version of ADM and compare the results in Table 3.

Solution Equation (19) is rewritten in the form:

$$
L_{t} u=x^{2} \sin ^{2} \frac{\pi t}{2}+\frac{\partial^{2} u}{\partial x^{2}}-\frac{\pi^{2}}{4} u-u^{2},
$$

where $N u=u^{2}$ and $L_{t} L_{t}=\frac{\partial^{2}}{\partial t^{2}}$.

Applying $L_{t}^{-1}()=.\int_{0}^{t} \int_{0}^{t}(.) \mathrm{d} t \mathrm{~d} t$ to (21), we get

$$
u=u(x, 0)+t u_{t}(x, 0)+L_{t}^{-1}\left(x^{2} \sin ^{2} \frac{\pi t}{2}\right)+L_{t}^{-1}\left(\frac{\partial^{2} u}{\partial x^{2}}\right)-L_{t}^{-1}\left(\frac{\pi^{2}}{4} u\right)-L_{t}^{-1}(N u) .
$$

Based on the recurrence relation (6) and substituting the initial value, we get

$$
\begin{aligned}
u_{0} & =\frac{\pi x t}{2}-\frac{x^{2}}{2 \pi^{2}}+\frac{x^{2}}{4}\left(t^{2}+\frac{2}{\pi^{2}} \cos (\pi t)\right), \\
u_{n+1} & =L_{t}^{-1}\left(\frac{\partial^{2} u_{n}}{\partial x^{2}}\right)-L_{t}^{-1}\left(\frac{\pi^{2}}{4} u_{n}\right)-L_{t}^{-1} A_{n}, n \geq 0,
\end{aligned}
$$

using El-Kalla polynomials formula (8), 


$$
\begin{aligned}
& \bar{A}_{0}=u_{0}^{2}, \\
& \bar{A}_{1}=2 u_{0} u_{1}+u_{1}^{2}, \\
& \bar{A}_{2}=2 u_{0} u_{2}+2 u_{1} u_{2}+u_{2}^{2} .
\end{aligned}
$$

Then, from (23) and (24) we get

$$
\begin{aligned}
u_{0}=\frac{\pi x t}{2} & -\frac{x^{2}}{2 \pi^{2}}+\frac{x^{2}}{4}\left(t^{2}+\frac{2}{\pi^{2}} \cos (\pi t)\right), \\
u_{1}= & -\frac{t^{2}}{2 \pi^{2}}+\frac{t^{4}}{24}+\frac{1-\cos (\pi t)}{\pi^{4}}-\frac{x\left(4 \pi^{5} t^{3}+24 x-12 \pi^{2} t^{2} x+\pi^{4} t^{4} x-24 x \cos (\pi t)\right)}{192 \pi^{2}} \\
& -\frac{1}{480 \pi^{6}} x^{2}\left(10 \pi^{8} t^{4}-240 \pi^{3} t x-40 \pi^{5} t^{3} x+6 \pi^{7} t^{5} x-945 x^{2}+90 \pi^{2} t^{2} x^{2}\right. \\
& -10 \pi^{4} t^{4} x^{2}+\pi^{6} t^{6} x^{2}-120 x\left(2 \pi^{3} t-8 x+\pi^{2} t^{2} x\right) \cos (\pi t) \\
& \left.-15 x^{2} \cos (2 \pi t)+480 \pi^{2} x \sin (\pi t)+480 \pi t x^{2} \sin (\pi t)\right) .
\end{aligned}
$$

$$
\begin{aligned}
& u_{2}=\frac{143}{8 \pi^{10}}+\frac{1}{2 \pi^{4}}-\frac{3 t^{2}}{4 \pi^{8}}-\frac{t^{2}}{4 \pi^{2}}+\frac{t^{4}}{48}+\frac{t^{4}}{12 \pi^{6}}-\frac{t^{6}}{90 \pi^{4}}-\frac{\pi^{2} t^{6}}{480}+\frac{t^{8}}{1344 \pi^{4}}-\frac{t^{10}}{51840} \\
& -\frac{18 \cos (\pi t)}{\pi^{10}}-\frac{\cos (\pi t)}{2 \pi^{4}}+\frac{4 t^{2} \cos (\pi t)}{\pi^{8}}-\frac{t^{4} \cos (\pi t)}{12 \pi^{6}}+\frac{\cos (2 \pi t)}{8 \pi^{10}}-\frac{12 t \sin (\pi t)}{\pi^{9}} \\
& +x\left(-\frac{41 t}{4 \pi^{5}}+\frac{t^{3}}{3 \pi^{3}}+\frac{5 t^{5}}{96 \pi}+\frac{\pi^{5} t^{5}}{3840}-\frac{11 \pi t^{7}}{3360}+\frac{\pi^{3} t^{9}}{41472}-\frac{19 t \cos (\pi t)}{4 \pi^{5}}+\frac{t^{3} \cos (\pi t)}{24 \pi^{3}}+\frac{15 \sin (\pi t)}{\pi^{6}}-\frac{t^{2} \sin (\pi t)}{4 \pi^{4}}\right) \\
& +x^{2}\left(-\frac{353}{4 \pi^{8}}-\frac{1}{32 \pi^{2}}+\frac{t^{2}}{64}+\frac{51 t^{2}}{4 \pi^{6}}-\frac{7 t^{4}}{768}+\frac{t^{6}}{45 \pi^{2}}+\frac{7 \pi^{4} t^{6}}{7680}-\frac{37 t^{8}}{26880}-\frac{\pi^{6} t^{8}}{129024}+\frac{\pi^{2} t^{10}}{41472}\right. \\
& \left.+\frac{177 \cos (\pi t)}{2 \pi^{8}}+\frac{\cos (\pi t)}{32 \pi^{2}}-\frac{8 t^{2} \cos (\pi t)}{\pi^{6}}+\frac{5 t^{4} \cos (\pi t)}{48 \pi^{4}}-\frac{\cos (2 \pi t)}{4 \pi^{8}}+\frac{49 t \sin (\pi t)}{\pi^{7}}-\frac{5 t^{3} \sin (\pi t)}{6 \pi^{5}}\right) \\
& +x^{3}\left(\frac{139 t}{8 \pi^{9}}+\frac{21 t}{32 \pi^{3}}-\frac{t^{3}}{12 \pi^{7}}+\frac{t^{5}}{60 \pi^{5}}-\frac{7 \pi t^{5}}{1280}+\frac{t^{7}}{630 \pi^{3}}+\frac{9 \pi^{3} t^{7}}{8960}-\frac{7 t^{9}}{25920 \pi}-\frac{5 \pi^{5} t^{9}}{331776}+\frac{\pi t^{11}}{105600}\right. \\
& +\frac{64 t \cos (\pi t)}{\pi^{9}}+\frac{23 t \cos (\pi t)}{32 \pi^{3}}-\frac{16 t^{3} \cos (\pi t)}{3 \pi^{7}}-\frac{5 t^{3} \cos (\pi t)}{192 \pi}+\frac{t^{5} \cos (\pi t)}{15 \pi^{5}}-\frac{t \cos (2 \pi t)}{8 \pi^{9}}-\frac{82 \sin (\pi t)}{\pi^{10}} \\
& \left.-\frac{11 \sin (\pi t)}{8 \pi^{4}}+\frac{24 t^{2} \sin (\pi t)}{\pi^{8}}+\frac{5 t^{2} \sin (\pi t)}{32 \pi^{2}}-\frac{3 t^{4} \sin (\pi t)}{4 \pi^{6}}+\frac{3 \sin (2 \pi t)}{8 \pi^{10}}\right) \\
& +x^{4}\left(\frac{2703479}{9216 \pi^{12}}+\frac{3811}{256 \pi^{6}}-\frac{95 t^{2}}{32 \pi^{10}}-\frac{45 t^{2}}{128 \pi^{4}}+\frac{83 t^{4}}{384 \pi^{8}}-\frac{5 t^{4}}{192 \pi^{2}}-\frac{13 t^{6}}{2880}-\frac{151 t^{6}}{11520 \pi^{6}}+\frac{13 t^{8}}{17920 \pi^{4}}\right. \\
& +\frac{151 \pi^{2} t^{8}}{258048}-\frac{11 t^{10}}{259200 \pi^{2}}-\frac{221 \pi^{4} t^{10}}{16588800}+\frac{t^{12}}{760320}-\frac{9415 \cos (\pi t)}{32 \pi^{12}}-\frac{477 \cos (\pi t)}{32 \pi^{6}}+\frac{723 t^{2} \cos (\pi t)}{8 \pi^{10}} \\
& +\frac{23 t^{2} \cos (\pi t)}{8 \pi^{4}}-\frac{85 t^{4} \cos (\pi t)}{24 \pi^{8}}-\frac{41 t^{4} \cos (\pi t)}{768 \pi^{2}}+\frac{t^{6} \cos (\pi t)}{40 \pi^{6}}+\frac{897 \cos (2 \pi t)}{1024 \pi^{12}}+\frac{5 \cos (2 \pi t)}{256 \pi^{6}} \\
& -\frac{39 t^{2} \cos (2 \pi t)}{512 \pi^{10}}+\frac{t^{4} \cos (2 \pi t)}{1536 \pi^{8}}-\frac{\cos (3 \pi t)}{288 \pi^{12}}-\frac{467 t \sin (\pi t)}{2 \pi^{11}}-\frac{159 t \sin (\pi t)}{16 \pi^{5}}+\frac{65 t^{3} \sin (\pi t)}{3 \pi^{9}} \\
& \left.+\frac{15 t^{3} \sin (\pi t)}{32 \pi^{3}}-\frac{23 t^{5} \sin (\pi t)}{60 \pi^{7}}+\frac{51 t \sin (2 \pi t)}{128 \pi^{11}}-\frac{t^{3} \sin (2 \pi t)}{384 \pi^{9}}\right)
\end{aligned}
$$




$$
\begin{aligned}
& +x^{5}\left(-\frac{39119 t}{2048 \pi^{7}}-\frac{53 t^{3}}{192 \pi^{5}}+\frac{47 t^{5}}{15360 \pi^{3}}-\frac{3 t^{7}}{1792 \pi}+\frac{29 \pi t^{9}}{138240}-\frac{17 \pi^{3} t^{11}}{2534400}-\frac{249 t \cos (\pi t)^{5}}{4 \pi^{7}}\right. \\
& +\frac{37 t^{3} \cos (\pi t)}{8 \pi^{5}}-\frac{5 t^{5} \cos (\pi t)}{96 \pi^{3}}+\frac{179 t \cos (2 \pi t)}{2048 \pi^{7}}-\frac{t^{3} \cos (2 \pi t)}{3072 \pi^{5}}+\frac{327 \sin (\pi t)}{4 \pi^{8}}-\frac{22 t^{2} \sin (\pi t)}{\pi^{6}} \\
& \left.+\frac{59 t^{4} \sin (\pi t)}{96 \pi^{4}}-\frac{249 \sin (2 \pi t)}{1024 \pi^{8}}+\frac{t^{2} \sin (2 \pi t)}{1024 \pi^{6}}\right) \\
& +x^{6}\left(-\frac{20131027}{73728 \pi^{10}}+\frac{411 t^{2}}{256 \pi^{8}}-\frac{511 t^{4}}{3072 \pi^{6}}+\frac{499 t^{6}}{92160 \pi^{4}}-\frac{457 t^{8}}{1290240 \pi^{2}}+\frac{103 t^{10}}{2073600}-\frac{61 \pi^{2} t^{12}}{30412800}\right. \\
& +\frac{70115 \cos (\pi t)}{256 \pi^{10}}-\frac{5663 t^{2} \cos (\pi t)}{64 \pi^{8}}+\frac{235 t^{4} \cos (\pi t)}{64 \pi^{6}}-\frac{9 t^{6} \cos (\pi t)}{320 \pi^{4}}-\frac{6917 \cos (2 \pi t)}{8192 \pi^{10}} \\
& +\frac{323 t^{2} \cos (2 \pi t)}{4096 \pi^{8}}-\frac{5 t^{4} \cos (2 \pi t)}{12288 \pi^{6}}+\frac{5 \cos (3 \pi t)}{2304 \pi^{10}}+\frac{3567 t \sin (\pi t)}{16 \pi^{9}}-\frac{175 t^{3} \sin (\pi t)}{8 \pi^{7}} \\
& \left.+\frac{199 t^{5} \sin (\pi t)}{480 \pi^{5}}-\frac{447 t \sin (2 \pi t)}{1024 \pi^{9}}+\frac{5 t^{3} \sin (2 \pi t)}{3072 \pi^{7}}\right) \\
& +x^{7}\left(\frac{1549169 t}{18432 \pi^{11}}-\frac{21 t^{3}}{64 \pi^{9}}-\frac{17 t^{5}}{1280 \pi^{7}}+\frac{229 t^{7}}{161280 \pi^{5}}-\frac{47 t^{9}}{82944 \pi^{3}}+\frac{t^{11}}{126720 \pi}-\frac{\pi t^{13}}{2995200}\right. \\
& +\frac{32631 t \cos (\pi t)}{64 \pi^{11}}-\frac{923 t^{3} \cos (\pi t)}{16 \pi^{9}}+\frac{121 t^{5} \cos (\pi t)}{80 \pi^{7}}-\frac{t^{7} \cos (\pi t)}{120 \pi^{5}}-\frac{2091 t \cos (2 \pi t)}{2048 \pi^{11}} \\
& +\frac{109 t^{3} \cos (2 \pi t)}{3072 \pi^{9}}-\frac{t^{5} \cos (2 \pi t)}{5120 \pi^{7}}+\frac{t \cos (3 \pi t)}{576 \pi^{11}}-\frac{4765 \sin (\pi t)}{8 \pi^{12}}+\frac{214 t^{2} \sin (\pi t)}{\pi^{10}}-\frac{263 t^{4} \sin (\pi t)}{24 \pi^{8}} \\
& \left.+\frac{7 t^{6} \sin (\pi t)}{48 \pi^{6}}+\frac{2817 \sin (2 \pi t)}{2048 \pi^{12}}-\frac{149 t^{2} \sin (2 \pi t)}{512 \pi^{10}}+\frac{t^{4} \sin (2 \pi t)}{1024 \pi^{8}}-\frac{\sin (3 \pi t)}{216 \pi^{12}}\right) \\
& +x^{8}\left(\frac{641700505}{884736 \pi^{14}}-\frac{12035 t^{2}}{4096 \pi^{12}}+\frac{63 t^{4}}{1024 \pi^{10}}-\frac{19 t^{6}}{3840 \pi^{8}}+\frac{41 t^{8}}{143360 \pi^{6}}-\frac{7 t^{10}}{518400 \pi^{4}}+\frac{t^{12}}{1520640 \pi^{2}}\right. \\
& -\frac{t^{14}}{41932800}-\frac{46525 \cos (\pi t)}{64 \pi^{14}}+\frac{33807 t^{2} \cos (\pi t)}{128 \pi^{12}}-\frac{465 t^{4} \cos (\pi t)}{32 \pi^{10}}+\frac{121 t^{6} \cos (\pi t)}{480 \pi^{8}}-\frac{t^{8} \cos (\pi t)}{960 \pi^{6}} \\
& +\frac{13609 \cos (2 \pi t)}{8192 \pi^{14}}-\frac{2119 t^{2} \cos (2 \pi t)}{4096 \pi^{12}}+\frac{109 t^{4} \cos (2 \pi t)}{12288 \pi^{10}}-\frac{t^{6} \cos (2 \pi t)}{30720 \pi^{8}}-\frac{17 \cos (3 \pi t)}{1728 \pi^{14}}+\frac{t^{2} \cos (3 \pi t)}{1152 \pi^{12}} \\
& +\frac{\cos (4 \pi t)}{32768 \pi^{14}}-\frac{4989 t \sin (\pi t)}{8 \pi^{13}}+\frac{1747 t^{3} \sin (\pi t)}{24 \pi^{11}}-\frac{263 t^{5} \sin (\pi t)}{120 \pi^{9}}+\frac{t^{7} \sin (\pi t)}{48 \pi^{7}}+\frac{2845 t \sin (2 \pi t)}{2048 \pi^{13}} \\
& \left.-\frac{149 t^{3} \sin (2 \pi t)}{1536 \pi^{11}}+\frac{t^{5} \sin (2 \pi t)}{5120 \pi^{9}}-\frac{t \sin (3 \pi t)}{216 \pi^{13}}\right) .
\end{aligned}
$$

Table 3 shows the absolute relative error (ARE) for the third-order approximate solution using the proposed version of ADM and the third-order approximate solution using the standard ADM at $t=0.2$ for some values of $x$ in Example 2. The exact solution of the partial differential Eq. (19) is given in [24] by $u(x, t)=x \sin \left(\frac{\pi t}{2}\right)$.

\section{Conclusion}

An accelerated technique based on ADM is proposed. In this proposed technique, there is no need for differentiation in calculations of the Adomian polynomials. Consequently, it makes programming easier and saves much time on the same processor compared with the calculations using traditional Adomian polynomials. Convergence analysis of this version is discussed, and the error analysis of the series solution is estimated. Results of numerical examples show the effectiveness of the proposed technique. Accordingly, in the future, this accelerated version is recommended for solving nonlinear equations with different complicated piece-wise differentiable nonlinearity terms.

\section{Abbreviations}

ADM: Adomian decomposition method; PDEs: Partial differential equations; NPDEs: Nonlinear partial differential equations; HAM: Homotopy analysis method; HPM: Homotopy perturbation method; ARE: Absolute relative error. 
Acknowledgements

Not applicable.

\section{Authors' contributions}

All authors (IE, EM, and $\mathrm{HE}$ ) contributed equally and significantly in writing this manuscript. All authors have read and approved the final manuscript.

\section{Funding}

Not applicable.

\section{Availability of data and materials}

Not applicable.

\section{Declarations}

\section{Competing interests}

The authors declare that they have no competing interest.

\section{Author details}

${ }^{1}$ Physics and Engineering Mathematics Department, Faculty of Engineering, Mansoura University, Mansoura, Egypt.

${ }^{2}$ Mathematics Department, Faculty of Science, Damietta University, New Damietta, Egypt.

Received: 27 June 2020 Accepted: 11 February 2021

Published online: 06 March 2021

\section{References}

1. Jafarimoghaddam, A.: On the Homotopy Analysis Method (HAM) and Homotopy Perturbation Method (HPM) for a nonlinearly stretching sheet flow of Eyring-Powel fluids. Eng. Sci. Technol. Int. J. 22(2), 439-451 (2019)

2. Matinfar, M., Saeidy, M., Khan, Y., Gharahsuflu, B.: Finding the exact solution of special nonlinear partial differential equations by homotopy analysis method. Walailak J Sci Technol. 11(3), 171-178 (2014). https://doi.org/10.2004/wjst. v11i3.310

3. Kurt, A., Tasbozan, O.: Approximate analytical solution of the time fractional Whitham-Broer-Kaup equation using the homotopy analysis method. Int. J. Pure Appl. Math. 98(4), 503-510 (2015)

4. Tasbozan, O., Kurt, A.: Approximate analytical solution of ZK-BBM equation. Sohag J. Math. 2(2), 57-60 (2015)

5. ul Haq, I.: Analytical approximate solution of non-linear problem by homotopy perturbation method. Matrix Sci. Math. (MSMK) 3(1), 20-24 (2019)

6. El-Sayed, A.M., Elsaid, A., El-Kalla, I.L., Hammad, D.: A homotopy perturbation technique for solving partial differential equations of fractional order in finite domains. Appl. Math. Comput. 218(17), 8329-8340 (2012)

7. Sanchez Cano, J.A.: Adomian decomposition method for a class of nonlinear problems. ISRN Appl. Math. (2011). https://doi.org/10.5402/2011/709753

8. El-Kalla, I.L.: Piece-wise continuous solution to a class of nonlinear boundary value problem. Ain Shams Eng. J. 4, 325-331 (2013)

9. El-Kalla, I.L., El Mhlawy, A.M., Botros, M.: A continuous solution of solving a class of nonlineartwo point boundary value problem using Adomian decomposition method. Ain Shams Eng. J. 10, 211-216 (2019)

10. Gaxiola, O.G., Jaquez, R.B.: Applying Adomian decomposition method to solve Burgess equation with a non-linear source. Int. J. Appl. Comput. Math. 3, 213-224 (2017)

11. Kaliyappan, M., Hariharan, S.: Solving nonlinear differential equations using Adomian decomposition method through Sagemath. Int. J. Innov. Technol. Explor. Eng. 8(6), 510-515 (2019)

12. El-Kalla, I.L.: New results on the analytic summation of Adomian series for some classes of differential and integral equations. Appl. Math. Comput. 217, 3756-3763 (2010)

13. El-Kalla, I.L.: A new approach for solving a class of nonlinear integro-differential equations. Commun. Nonlinear Sci. Numer. Simul. 17, 4634-4641 (2012)

14. Gundoğdu, H., Gzukızıl, O.F.: Solving nonlinear partial differential equations by using Adomian decomposition method, modified decomposition method and Laplace decomposition method. MANAS J. Eng. 5(1), 1-13 (2017)

15. Alhaddad, S.M.: Adomian decomposition method for solving the nonlinear heat equation. Int. J. Eng. Res. Appl. 7, 97-100 (2017)

16. Duan, J.S.: New recurrence algorithms for the nonclassic Adomian polynomials. Comput. Math. Appl. 62(8), 2961-2977 (2011a)

17. Duan, J.S.: Recurrence triangle for Adomian polynomials. Appl. Math. Comput. 216, 1235-1241 (2010)

18. Duan, J.S.: New recurrence algorithm for the nonclassic Adomian polynomials. Comput. Math. Appl. 62, 2961-2977 (2011b)

19. Duan, J.S., Rach, R.: Higher-order numeric Wazwaz-El-Sayed modified Adomian decomposition algorithms. Comput. Math. Appl. 63, 1557-1568 (2012)

20. Zaouagui, I.N., Badredine, T.: New Adomian's polynomials formulas for the non-linear and nonautonomous ordinary differential equations. J. Appl. Comput. Math. (2017). https://doi.org/10.4172/2168-9679.100073

21. El-Kalla, I.L.: Error analysis of Adomian series solution to a class of nonlinear differential equations. Appl. Math. E-Notes 7, 214-221 (2007)

22. Behiry, S.H., Hashish, H., El-Kalla, I.L., Elsaid, A.: A new algorithm for the decomposition solution of nonlinear differential equations. Comput. Math. Appl. 54, 459-466 (2007) 
23. El-Kalla, I.L., Abd Elgaber, K.M., Elmahdy, A.R., Sayed, A.Y.: Solution of a nonlinear delay differential equation using Adomian decomposition method with accelerated formula of Adomian polynomial. Am. J. Comput. Math. 9, 221-233 (2019)

24. Basak, K.C., Ray, P.C., Bera, R.K.: Solution of non-linear Klein-Gordon equation with a quadratic non-linear term by Adomian decomposition method. Commun. Nonlinear Sci. Numer. Simul. 14, 718-723 (2009)

\section{Publisher's Note}

Springer Nature remains neutral with regard to jurisdictional claims in published maps and institutional affiliations.

Submit your manuscript to a SpringerOpen ${ }^{\circ}$ journal and benefit from:

- Convenient online submission

- Rigorous peer review

- Open access: articles freely available online

- High visibility within the field

Retaining the copyright to your article

Submit your next manuscript at $>$ springeropen.com 